\title{
INTERVISTA A FABIO PIERANGELI
}

Maria Franca Zuccarello ${ }^{1}$

Jefferson Evaristo do Nascimento Silva²

Fabio Pierangeli, nato a Roma nel 1962, è Professore Associato del gruppo scientifico-disciplinare di Lingua, Filologia e Letterature, presso la Facoltà di Lettere e Filosofia dell'Università degli Studi di Roma "Tor Vergata". I suoi studi si concentrano principalmente sulla tematica del secolo XX, essendo lui il maggior ricercatore del mondo di Cesare Pavese e Italo Calvino. Attualmente, è il Coordinatore del Dottorato Internazionale di Letteratura Comparata dell'Università Tor Vergata, dove insegna ed è il Tutor di diversi studenti. Tra i suoi principali libri, abbiamo Pavese e i suoi miti toccati dal destino. Per una lettura di Dialoghi con Leucò; Italo Calvino, la metamorfosi e l'idea del nulla; Una luce particolare, non so come descriverla; Luoghi e viaggi nella letteratura contemporânea; Destini e identità romane da D'Annunzio ai contemporanei ed altri. L'anno scorso, ad agosto, è stato alla UERJ per fare una conferenza sulla cultura italiana, nell'ambito dell'anno Italia-Brasile. Ho avuto modo di starci un po' insieme - e devo dire que è una persona bellissima da conoscere! - ed abbiamo pensato di fare quest'intervista, con l'obiettivo di farlo conoscere in Brasile. Siccome lui era ritornato in Italia, l'abbiamo fatta per Internet - il che non ha tolto niente alla sua importanza. È il nome giusto per tutti quelli che vogliono comprendere ed imparare la letteratura italiana, critico di lettura obbligatoria, ci fa regalo di alcune parole sulla sua vita, studi e visione. Vediamole:

\footnotetext{
${ }^{1}$ Professora Adjunta do Instituto de Letras/ Dept ${ }^{\mathrm{o}}$ Letras Neolatias/ Setor de Italiano da Universidade do Estado do Rio de Janeiro (e-mail: mfrancazuccarello@ superig.com.br).

${ }^{2}$ Bolsista de Iniciação à Docência / UERJ (e-mail: jeffersonpn@yahoo.com.br).
} 
RIU: Il piú noto romanzo di Cesare Pavese, La luna ed i falo, scritto poco tempo prima di suicidarsi, è pura autobiografia. Quali sono i principali temi da lui affrontati e fra tutti qual è quello che rispecchia di più il suo carattere?

F. Pierangeli: Pura autobiografia, certo, ma trasfigurata attraverso gli strumenti propri della letteratura, con una tecnica narrativa diventata sorvegliatissima, eppure, per così dire naturale. Pavese scrive nel Diario di aver presentito i contenuti della Luna e i falò fin dall'inizio del suo farsi scrittore e di averlo scritto il soli tre mesi, con una incredibile gioia creativa. Rimane evidente che quella di Pavese è una poetica circolare, monotona, come lui stesso la definisce: battendo e ribattendo sempre sullo stesso grumo esistenziale, sugli stessi temi, il viaggio, la fuga, il ritorno, il mito, la durezza del lavoro, la guerra come esplosione di una carattere indelebile dell'umanità, il male e la violenza. Stessa situazione all'inizio e alla fine della carriera artistica dello scrittore, lungo vent'anni: dal 1930, I Mari del Sud, al 1949-1950 La luna e i falò. Due personaggi, il ragazzino e il Cugino nella lirica, Nuto e Anguilla nel romanzo, ascendono la collina e dialogano sulle profonde verità dell'esistenza, sulla storia umana. Naturalmente con più coscienza, nel 1950, soprattutto per l'infuriare della Seconda Guerra Mondiale, passata con particolare violenza in quelle colline, con la "guerra civile" tra partigiani e fascisti. Il tema principale è quello di un inutile andare per il mondo a cercare quello che è scritto nel paesetto che si è lasciati, nel volto di persone che non ci sono più, di fronte al male che sempre si registra nella storia degli uomini. Il falò ci riporta a miti ancestrali, in cui la violenza e il sangue erano, come nel presente, e come sempre, una forza irrazionale e quasi necessaria, nonostante l'ideologia buona, quella comunista, rappresentata da Nuto, che ha ancora voglia di cambiare il mondo. Come un falò brucia la casa del Valino, impazzito a causa del lavoro bestiale che deve sopportare, nel falò, alla fine del romanzo, brucia il corpo di Santina, il simbolo dolce di un tempo andato, quello dell' infanzia, che non può tornare. Pavese si sente sradicato, come Anguilla, anche se ammira quel tempo lontano che coincideva con il tempi della natura "quando tutto si faceva a stagione. Un tempo ordinato e quasi liturgico, che Pavese, nel suo modo secco, stringente e realista, riproduce, in certi passaggi quasi biblicamente. Dietro le vicende degli uomini, insomma, dietro e dentro il destino, c'è una realtà altra, ben descritta dai miti ancestrali. Pavese ha 
raggiunto splendidamente il suo obiettivo di scrittore: unire il piglio vivace del realismo con la "profondità di sensi del simbolismo".

RIU: Qual è il motivo per cui Pavese scrive un rimanzo con cui ripercorre con la memorialuoghi, persone, e tutto ciò che si riferisce alla sua propria infanzia?

F. Pierangeli: Lo sente dentro profondamente, con estrema urgenza. Quello è il suo mondo e deve descriverlo, qualcosa che gli è dentro. Si legga anche il dialogo L'isola, nei Dialoghi con Leucò, o La langa, in Feria d'Agosto, legati all'identico tema.

RIU: Cosa vuol significare il rapporto di Anguilla con il piccolo Cinto? Come si configura il rapporto tra Nuto e il protagonista e quali sono i motivi che stanno alla base del loro legame?

F. Pierangeli: Siamo di fronte ad un quadilatero molto interessante: Anguilla vuole essere una specie di guida per Cinto, rivedendosi in lui. Anguilla è un bastardo, ma ha fatto fortuna in America, Cinto non si è mosso da quel paese e vive una condizione sociale estremamente drammatica, fino all'episodio dell'incendio della casa del Valino. Il futuro per lui è come bloccato da queste tragedie: Pavese riesce nell' intento di descrivere e denunciare una situazione reale, come lo sfruttamento del lavoro minorile, e nello stesso tempo proporci una visione più universale, esistenziale attraverso quelle figure. Per Anguilla, viceversa, è Nuto la guida, colui che rimanendo attaccato a quel paese, alla terra, alla natura, non vive l'inquietudine dell'amico più giovane. Anzi, abbraccia con energia positiva, pur continuando a credere alle "superstizioni del mondo contadino, il comunismo, nella sua dinamica più felice: la vicinanza vera al popolo, alle sue miserevoli condizioni sociali. Eppure anche Nuto non può che raccontare l'episodio di Santina, con grande commozione. Con la tragedia della guerra si sono ripetute con ferocia le situazioni di violenza e di guerra della storia dell'uomo.

RIU: La posizione in cui è ambientato il contesto Lei pensa sia frutto della realtà o d'invenzione dell'Autore?

F. Pierangeli: Sicuramente di una realtà storica ben precisa che, come dicevo, Pavese trasfigura anche a livello simbolico e universale. I fatti che descrive gli sono stati 
veramente raccontati da Nuto, Pinolo Scaglione, in lunghe passeggiate tra le colline.

RIU: Italo Calvino è conosciuto per la sua trilogia: cosa si proponeva di mostrare con i personaggi protagonisti? A chi erano indirizzati i suoi "commenti" sulla morale e sull'ideologia?

F. Pierangeli: La forte componente ideologica del giovane Calvino, ispirata da Gramsci e altri autori, si unisce in lui, fin dall' inizio ad una forte tensione fantastica: nascono allora libri particolarissimi come Il sentiero dei nidi di ragno, che vede la guerra di resistenza con gli occhi di un bambino, accolta in una banda partigiana scalcagnata e fuori dalle regole militari.

RIU: La trilogia di Italo Calvino, riassunta poi in I Nostri Antenati, è una lettura degli anni di gioventù il cui ricordo si era perso nel passar del tempo insieme alla massa enorme delle nostre dimenticanze. Oggi si dice in Italia che Calvino sia se non il maggiore almeno uno fra i maggiori letterati italiani della seconda metà del ventesimo secolo. Una rilettura delle sue opere, a cominciare da questi tre romanzi, può o non può dirci se il giudizio dei posteri è meritatamente giusto o ancora affrettato?

F. Pierangeli: Per fortuna il campo della letteratura e dei gusti letterari è vastissimo e complesso. Sicuramente Calvino, con Pavese, Gadda, Moravia e alcuni altri è tra gli autori più importanti della seconda metà del Novecento italiano.

RIU: Si è detto che "non è facile giudicare uno scrittore complesso e mutevole come Italo Calvino, che all'inizio della sua carriera di scrittore si gridò al miracolo, al fenomeno letterario. Altri, forse meno attrezzati intellettualmente, rimasero freddi e lo dimenticarono subito, appena terminata la lettura di libri che erano stati portati alla ribalta dalla moda del momento. Altri ancora, in genere appartenenti alla generazione che alla fine degli anni sessanta aveva vent'anni, si appassionarono alla scoperta delle sue varie opere, anche se ad un certo momento alcuni non riuscirono a valicare l'ostacolo maggiore della lettura di Le Cosmocomiche, libro impervio e intellettualizzante di non facile assimilazione". 
Lei come lo giudica, e come giudica questi commenti diretti ad un autore che ha dominato, per diversi decenni ha dominato la scena letteraria italiana? F. Pierangeli: Calvino ha saputo attraversare diverse stagioni letterarie, cambiando spesso volto, ma rimanendo coerente ad una visione dello stile come leggerezza. Aveva, al contrario degli espressionisti, la volontà di togliere peso, almeno nella scrittura, alla materia. La sua evoluzione verso una zona totalmente fantastica, pur con precisi giudizi sulla realtà, a ben guardare, è un motivo importante della letteratura che giudico in modo assolutamente positivo.

RIU: Qual è il messaggio che il Calvino vuol dare alla società con la trilogia dei suoi romanzi brevi, Il visconte dimezzato, Il barone rampante e Il cavaliere inesistente?

F. Pierangeli: In anni di Guerra fredda, Calvino rappresenta la crisi sociale e esistenziale dell'uomo ricorrendo al favoloso: il suo scegliere mondi lontani contiene un ben preciso messaggio di pace, leggerezza, tensione umanistica verso il bello che la scrittura predilige.

RIU: Ad una domanda fatta a Calvino sulla trilogia lui risponde: «Siete padroni di interpretare come volete queste tre storie e non dovete sentirvi vincolati dalla deposizione che ora ho reso della loro genesi, scrive l'autore. Ho voluto farne una trilogia di esperienze sul come realizzarsi come esseri umani: ne Il Cavaliere inesistente la conquista dell'essere, ne Il Visconte dimezzato l'aspirazione a una completezza al di la delle mutilazioni imposte dalla società, ne Il Barone rampante una via verso una completezza non individualistica da raggiungere attraverso la fedeltà ad un'autodeterminazione individuale: tre gradi d'approcio alla libertà. E nello stesso tempo ho voluto che fossero tre storie, come si dice, aperte, che innanzitutto stiano in piedi come storie, per la logica del succedersi delle loro immagini, ma che comincino la loro vera vita nell'imprevedibile gioco d'interrogazioni e risposte suscitate nel lettore. Vorrei che potessero essere guardate come un albero genealogico degli antenati dell'uomo contemporaneo, in cui ogni volto cela qualche tratto delle persone intorno, di voi, di me stesso». E Lei, cosa ne pensa? 
F. Pierangeli: Sicuramente si tratta di un messaggio di libertà. Ma Calvino ha bisogno di uno sguardo a distanza, di riflettere sulle cose, di non affrontarle di "petto": deve salire sugli alberi e guardare dall'alto!

RIU: A proposito di "Se una notte d'inverno un viaggiatore" Cesare Segre dice che "è un «romanzo sulla teoria del romanzo", il cui vero protagonista è lo Scrittore, che mette in mostra i suoi meccanismi, giocando abilmente con le strutture linguistiche e narrative, Dentro queste strutture si riflette, sebbene mai definitivamente rappresentata, la continuità della vita che, densa delle sue realtà empiriche, è sempre il fine della tensione narrativa. Lo scrittore approderà così al suo testamento umano e artistico nelle prose di Palomar, nelle quali si ríconferma questa volontà di narrare, perché «se il tempo deve finire, lo si può descrivere istante per istante e ogni istante, a descriverlo, si dilata tanto che non se ne vede più la fine». Lei è d'accordo con questo?

F. Pierangeli: Certamente: Calvino ci offre una romanzo molto divertente sui meccanismi stessi del narrare, componendo una cornice classica e dieci primi capitoli di racconti tutti corrispondenti ai generi letterari più in voga. Palomar è il testamento letterario di Calvino, scrittore che non vuole smettere di cercare e di meravigliarsi. Ma la sua ricerca parte dal basso, dai fenomeni, sempre la presunzione di avere una idea assoluta. E ce ne sono alcune domande che ne penso che siano meno importante, ma che anche ti invio.

RIU: Professore, può raccontarci un po' della tua traiettoria con la letteratura? E perchè ha scelto Pavese e Calvino specificamente?

F. Pierangeli: Direi che non scegliamo, ma siamo scelti. Pavese per una copertina un poco "piccante" che illustrava la Bella estate negli Oscar Mondadori, Naturalmente era così per un adolescente. Da allora Pavese, per tutt'altre ragione, è rimasto per autore centrale, per la nuda sincerità con cui espone i temi centrali della letteratura e, quindi, della vita. 
RIU: Com'è l'esperiencia di insegnare letteratura comparata a stranieri? $\mathrm{E}$ quella di coordinare il dottorato di letteratura comparata internazionale della "Tor Vergata"?

F. Pierangeli: Sono esperienza che sono appena iniziate e tutto è ancora da costruire. Intanto insegnare per qualche ora ai dottorandi di italiano di Santa Caterina, grazie all'Amicizia di Patrizia Peterle è stato affascinante: ho trovato allievi molto preparati. Ho imparato molto.

RIU: Calvino fu anche un gran attivista politico nel suo tempo. Come ne vedi l'influenza di queste attività nel lavoro di Calvino?

F. Pierangeli: Influenza nel lavoro editoriale direi decisiva. In quella creativa Calvino ha vissuto, credo, con inquietudine e serietà una vocazione al racconto d'avventura e fantastico, cercando di scrivere anche romanzi impegnati e d'attualità, senza troppo successo, a parte delle ottime eccezioni come La speculazione edilizia e La giornata d'uno scrutatore, il suo libro, da questo punto di vista, più sincero.

RIU: Calvino sempre ha saputo ricercare e lavorare nelle diverse scuole letterarie, passando del neorealismo fino al postmodernismo. Come si può analizzare queste influenze nella sua scritta e, sesi può dire così, in quale scuola lui si sentiva più "a casa" e dove ha lasciato i suoi principali lavori?

F.Pierangeli: Si trovava a casa nell'idea di una letteratura che aiuti il mondo a guardare alla bellezza, contro il disordine e la violenza.

RIU: Pavese fu, secondo il proprio Calvino, il suo "primo lettore". Che tipo di influenza uno ha acquisito dell'altro?

F. Pierangeli: Proprio Cesare Pavese salutava, con una recensione da grande Maestro, il primo libro, uscito nel 1947, di quel giovanissimo collaboratore della Casa editrice Einaudi. Nato a Cuba nel 1922, da genitori sanremesi, Calvino si era trovato al centro della grande cultura italiana studiando nella "Torino operosa" dei Gramsci e dei Gobetti e appunto, di Cesare Pavese. Quasi profeticamente intuendo le gesta del Barone rampante, lo scrittore langarolo scriveva del Sentiero dei nidi di ragno e della capacità di Calvino di 
descrivere la Resistenza con toni alati: «Diremo allora che l'astuzia di Calvino, scoiattolo della penna, è stata questa di arrampicarsi sulle piante, più per gioco che per paura e osservare la vita partigiana come un favola di bosco, clamorosa, variopinta, diversa». In quel momento dell'immediato dopoguerra (dopo aver vissuto rocambolesche esperienze personali intorno all'8 settembre, datosi partigiano, catturato, riuscito fortunosamente a fuggire ai tedeschi), la spinta fantastica coincide perfettamente con l'ideologia comunista di Calvino, destinata, è noto, ad incrinarsi progressivamente dopo i fatti di Ungheria e Polonia del '56-'57 e che lo porteranno anche a diversissime ricerche letterarie. Come è stato per Pavese, vede nei compagni i tipi più impegnati, vivaci e risoluti nella ricostruzione del Paese. La realtà così intravista mantiene lo stesso fascino e il ritmo dei grandi romanzi d'avventura che avevano infiammato la sua adolescenza: Conrad su tutti. Scrive ancora Pavese: «Bella la favola dei nidi di ragno. Piena di vita sobria e fantastica la lingua che costruisce questo mondo di bruttura e malizia e giochi. E' senza dubbio il primo racconto che a mio parere faccia poesia dell'esperienza partigiana, e ciò per virtù anzitutto del punto di vista - l'avventura del ragazzo». Pavese aveva perfettamente capito la vocazione di Calvino e lo instrada su quella. Calvino, dopo alcuni articoli sul suo Maestro, se ne dimentica: volontariamente. La tragica visione del mondo di Pavese non gli appartiene. Calvino, con le armi di Perseo, vuole sfuggire allo sguardo paralizzante di Medusa. Pavese la guarda negli occhi, fino all'estremo duello. «Verrà la morte e avrà i tuoi occhì. 